\title{
On the Computational Complexity of Cut-Elimination in Linear Logic
}

\author{
Harry G. Mairson ${ }^{1}$ and Kazushige Terui ${ }^{2}$ \\ 1 Computer Science Department, Brandeis University, \\ Waltham, Massachusetts 02454, USA \\ mairson@cs.brandeis.edu \\ 2 National Institute of Informatics, 2-1-2 Hitotsubashi, Chiyoda-ku, \\ 101-8430 Tokyo, Japan \\ terui@nii.ac.jp
}

\begin{abstract}
Given two proofs in a logical system with a confluent cutelimination procedure, the cut-elimination problem (CEP) is to decide whether these proofs reduce to the same normal form. This decision problem has been shown to be PTIME-complete for Multiplicative Linear Logic (Mairson 2003). The latter result depends upon a restricted simulation of weakening and contraction for boolean values in MLL; in this paper, we analyze how and when this technique can be generalized to other MLL formulas, and then consider CEP for other subsystems of Linear Logic. We also show that while additives play the role of nondeterminism in cut-elimination, they are not needed to express deterministic PTIME computation. As a consequence, affine features are irrelevant to expressing PTIME computation. In particular, Multiplicative Light Linear Logic (MLLL) and Multiplicative Soft Linear Logic (MSLL) capture PTIME even without additives nor unrestricted weakening. We establish hierarchical results on the cut-elimination problem for MLL(PTIME-complete), MALL(coNP-complete), MSLL(EXPTIME-complete), and for MLLL (2EXPTIME-complete).
\end{abstract}

\section{Introduction}

Cut-elimination is naturally seen as a function from proofs to their normal form, and we can derive from it an equally natural decision problem: if $L$ is a logical system with a confluent cut-elimination procedure, and we are given two proofs in $L$, do they reduce to the same normal form? Call this the cut elimination problem (CEP). When $L$ has reasonable representations of boolean values as proofs, an even simpler decision problem is to ask: given a proof, does it reduce to the representation for "true"?

Through the Curry-Howard correspondence, we know that proofs in linear logics represent programs, typically in a functional programming langauge with highly specified forms of copying, where cut-elimination serves as an interpreter: normalization is evaluation. The cut-elimination problem is then a fundamental question about program equivalence, and how hard it is to decide. Moreover, the 
correspondence facilitates our identification of particular logics with associated complexity classes, where our goal is to link the expressive power of proofs with a suitably powerful interpreter that can "run" representations of programs in that complexity class.

The cut-elimination problem is known to be non-elementary for simply typed $\lambda$-calculus [Sta79], and hence for linear logic. Low order fragments of simply typed $\lambda$-calculus are investigated in [Sch01]. In this paper, we consider the decision problem for various weak subsystems of linear logic that have no exponentials, or have very weak forms of them (i.e., the so-called "light" linear logics). Such an investigation suggests another way to characterize the complexity of linear logics: not only by the complexity of theorem proving (proof search)see, for example, [Lin95] — but also by the complexity of theorem simplification (proof normalization).

Even in intuitionistic multiplicative linear logic (IMLL), which has no exponentials, it is possible to simulate weakening and contraction for a restricted set of formulas, including a formula whose proofs code boolean values. As a consequence, we derive PTIME-completeness for CEP in IMLL; see Section 2. This result contradicts folkloric intuitions that MLL proofnets could be normalized in logarithmic space - that is, with only a finite number of pointers into the proofnet, presumably following paths in the style of the geometry of interaction. Similar to the results for IMLL, in Section 3 we derive conP-completeness results for IMALL, where we also have additives.

An alternative way to represent a complexity class by some logic is to consider the functions realizable (say, by a Turing machine) in the class, and show how each can be coded as a fixed proof (program) in the logic. For example, Light Linear Logic has been shown to so represent PTIME computations [Gir98], and the use of additives in that proof was replaced by unrestricted weakening in Light Affine Logic [Asp98,AR02]. We improve these results to show that such weakening is also unnecessary: Multiplicative Light Linear Logic is sufficient to capture PTIME (see Section 4), where we also prove that deciding CEP is complete for doubly-exponential time. Finally, in Section 5 we show similar characterizations of exponential time in Multiplicative Soft Linear Logic [Laf01].

\section{Expressivity of Multiplicatives}

\subsection{Weakening in MLL}

We restrict our attention to the intuitionistic $(-\circ, \forall)$ fragment IMLL of MLL, although all the results in this section carry over to the full classical MLL with no difficulty. Moreover, we omit type annotation from the proof syntax, and identify proofs of IMLL with type-free terms of linear $\lambda$-calculus.

A term (proof) of IMLL is either a variable $x$, or an application $(t u)$ where $t$ and $u$ are terms such that $F V(t) \cap F V(u)=\emptyset$, or an abstraction $(\lambda x . t)$ where $t$ is a term and $x \in F V(t)$. Terms are considered up to $\alpha$-equivalence, and the variable convention is adopted. The substitution operation $t[u / x]$ and the $\beta$ reduction relation are defined as usual. The size $|t|$ of a term $t$ is the number of 
nodes in its syntax tree. The type assignment rules are as follows ${ }^{3}$ :

$$
\begin{array}{ccc} 
& \frac{\Gamma \vdash u: A \quad x: A, \Delta \vdash t: C}{\Gamma, \Delta \vdash t[u / x]: C} & \frac{x: A, \Gamma \vdash t: B}{\Gamma \vdash \lambda x . t: A \multimap B} \\
\hline x: A \vdash x: A & \frac{\Gamma \vdash t: A}{\Gamma \vdash t} \alpha \notin F V(\Gamma) & \frac{x: A[B / \alpha], \Gamma \vdash t: C}{x: \forall \alpha . A, \Gamma \vdash t: C} \\
\frac{\Gamma \vdash u: A}{\Gamma, y: A \multimap B, \Delta \vdash t[y u / x]: C} & \frac{\Gamma \vdash t: \Delta \cdot A}{\Gamma \vdash t}
\end{array}
$$

Here, $\Gamma, \Delta \ldots$ stand for finite multisets of declarations $x: A$ and $F V(\Gamma)$ denotes the set of all free type variables in $\Gamma$. We say that a term $t$ is of type $A$ (or $t$ is a proof of $A$ ) if $\vdash t: A$ is derivable by the above rules. A type $A$ is inhabitied if there is a term of type $A$.

Unit 1 and tensor product $\otimes$ are introduced by means of the second order definitions:

$$
\begin{array}{rlrl}
\mathbf{1} & \equiv \forall \alpha . \alpha \multimap \alpha & A \otimes B & \equiv \forall \alpha .(A \multimap B \multimap \alpha) \multimap \alpha \\
\mathrm{I} & \equiv \lambda x \cdot x & t \otimes u & \equiv \lambda x . x t u
\end{array}
$$

let $t$ be I in $u \equiv t u \quad$ let $t$ be $x \otimes y$ in $u \equiv t(\lambda x y . u)$.

Tensor product is naturally extended to $n$-ary ones: $t_{1} \otimes \ldots \otimes t_{n}$ and let $u$ be $x_{1} \otimes$ $\cdots \otimes x_{n}$ in $t$. The expression $\lambda x_{1} \otimes \cdots \otimes x_{n}$.t stands for $\lambda z$.let $z$ be $x_{1} \otimes \cdots \otimes x_{n}$ in $t$. We also use shorthand notations such as $i d \equiv \lambda x . x, t \circ u \equiv \lambda x . t(u(x)), A^{n} \equiv$ $\underbrace{A \otimes \cdots \otimes A}_{n \text { times }}, A^{(n)} \multimap B \equiv \underbrace{A \multimap \cdots A \multimap}_{n \text { times }} B$.

Our first observation is that a version of weakening rule can be constructed for a certain restricted class of IMLL formulas.

Definition $1\left(\Pi_{1}, \Sigma_{1}, e \Pi_{1}, e \Sigma_{1}\right) . A$ type $A$ is $\Pi_{1}\left(\Sigma_{1}\right)$ if it is built from type variables by $-\mathbf{1}, \otimes$ (viewed as primitives) and positive (negative) occurrences of $\forall$. An e $\Pi_{1}\left(e \Sigma_{1}\right)$ type is like a $\Pi_{1}\left(\Sigma_{1}\right)$ type, but it may additionally contain negative (positive) occurrences of inhabited $\forall$-types.

The above definition of $\Pi_{1}$ and $e \Pi_{1}$ involves 1 and $\otimes$ as primitives, but we may ignore them in practice, because negative occurrences of $\otimes$ and $\mathbf{1}$ can be removed by isomorphisms $((A \otimes B) \multimap C) \circ-\circ(A \multimap B \multimap C)$ and $(\mathbf{1} \multimap C) \circ-\circ C$, while positive occurrences can be replaced with their $\Pi_{1}$ definitions.

Finite data types are naturally represented by closed inhabited $\Pi_{1}$ types. A typical example is the boolean type: $\mathbf{B} \equiv \forall \alpha . \alpha \multimap \alpha \multimap \alpha \otimes \alpha$. Meanwhile, functional types over those finite data types, such as $(\mathbf{B} \multimap \mathbf{B}) \multimap \mathbf{B}$, are all included in the class $e \Pi_{1}$.

Theorem 1 (e $\Pi_{1}$-Weakening). For any closed $e \Pi_{1}$ type $A$, there is a term $\mathrm{w}_{A}$ of type $A \multimap \mathbf{1}$.

\footnotetext{
${ }^{3}$ Note that any term of linear $\lambda$-calculus has a propositional type [Hin89]; the role of second order quantifers is not to increase the number of typable terms, but to assign a uniform type to structurally related terms.
} 
Proof. Without loss of generality, we may assume that $A$ does not contain $\otimes$ and $\mathbf{1}$. Let $B[\mathbf{1}]$ be the type $B$ with all free variables replaced with $\mathbf{1}$. By simultaneous induction, we prove: (i) for any $e \Pi_{1}$ type $B, B[\mathbf{1}] \vdash \mathbf{1}$ is provable; and (ii) for any $e \Sigma_{1}$ type $B, \vdash B[\mathbf{1}]$ is provable. When $B$ is a variable, the claims are obvious. When $B$ is $C \multimap D$, for (i) we derive $(C \multimap D)[\mathbf{1}] \vdash \mathbf{1}$ from $\vdash C[\mathbf{1}]$ and $D[\mathbf{1}] \vdash \mathbf{1}$, and for (ii) we derive $\vdash(C \multimap D)[\mathbf{1}]$ from $C[\mathbf{1}] \vdash \mathbf{1}$ and $\vdash D[\mathbf{1}]$. Let $B$ be $\forall \alpha$. $C$. If $B$ is $e \Pi_{1}$, we derive $(\forall \alpha . C)[\mathbf{1}] \vdash \mathbf{1}$ from $C[\mathbf{1}] \vdash \mathbf{1}$. If $B$ is $e \Sigma_{1}, \vdash B$ is provable by definition, and so is $\vdash B[\mathbf{1}]$.

\subsection{Encoding boolean circuits}

Let $A$ be an arbitrary type, and $B$ be a type that supports weakening in the sense we have just described; we can then define a projection function $\mathrm{fst}_{B}$ : $A \otimes B \multimap A$, given by fst $\mathrm{ft}_{B} \equiv \lambda x$. let $x$ be $y \otimes z$ in (let $\mathrm{w}_{B}(z)$ be $\mathrm{I}$ in $y$ ). By using this coding, we can then specify boolean values, weakening, and operations (including duplication) as:

$$
\begin{array}{ll}
\text { true } \equiv \lambda x y \cdot x \otimes y & : \mathbf{B} \\
\text { false } \equiv \lambda x y \cdot y \otimes x & : \mathbf{B} \\
\left.\mathrm{w}_{\mathbf{B}} \equiv \lambda z . \text { let } z l l \text { be } x \otimes y \text { in (let } y \text { be I in } x\right) & : \mathbf{B} \multimap \mathbf{1} \\
\text { not } \equiv \lambda P x y . P y x & : \mathbf{B} \multimap \mathbf{B} \\
\text { or } \equiv \lambda P Q . f^{\prime} t_{\mathbf{B}}(P \text { true } Q) & : \mathbf{B} \multimap \mathbf{B} \multimap \mathbf{B} \\
\text { cntr } \equiv \lambda P . \text { fst }_{\mathbf{B} \otimes \mathbf{B}}(P(\text { true } \otimes \text { true })(\text { false } \otimes \text { false })) & : \mathbf{B} \multimap \mathbf{B} \otimes \mathbf{B}
\end{array}
$$

Recall that a language $X \subseteq\{0,1\}^{*}$ is logspace reducible to $Y \subseteq\{0,1\}^{*}$ if there exists a logspace function $f:\{0,1\}^{*} \longrightarrow\{0,1\}^{*}$ such that $w \in X$ iff $f(w) \in Y$. Language $X$ is PTIME-complete if $X \in$ PTIME and each language $L \in$ PTIME is logspace reducible to $X$; a decision problem is said to be PTIMEcomplete when the language defined by that problem is PTIME-complete. The canonical PTIME-complete decision problem is the following:

Circuit Value Problem: Given a boolean circuit $C$ with $n$ inputs and 1 output, and truth values $\boldsymbol{x}=x_{1}, \ldots, x_{n}$, is $\boldsymbol{x}$ accepted by $C$ ? [Lad75]

Using the above coding of boolean operations, the problem is logspace reducible to CEP for IMLL:

Theorem 2 (PTIME-completeness of IMLL, [Mai03]). There is a logspace algorithm which transforms a boolean circuit $C$ with $n$ inputs and $m$ outputs into a term $t_{C}$ of type $\mathbf{B}^{n} \multimap \mathbf{B}^{m}$, where the size of $t_{C}$ is $O(|C|)$. As a consequence, the cut-elimination problem for IMLL is PTIME-complete.

Since binary words of length $n$ can be represented by $\mathbf{B}^{n}$, any finite function $f:\{0,1\}^{n} \longrightarrow\{0,1\}^{m}$ can be represented by a term $t_{f}: \mathbf{B}^{n} \multimap \mathbf{B}^{m}$. In this sense, MLL captures all the finite functions. 


\subsection{Contraction in MLL}

One of the key observations in proving Theorem 2 is that contraction is available for $\mathbf{B}$. We now generalize this observation, and show that the same holds for all closed inhabited $\Pi_{1}$ types (i.e. finite data types). First we show that conditional is available in IMLL:

Lemma 1 (Conditional). Let $t_{1}$ and $t_{2}$ be terms such that $x_{1}: C_{1}, \ldots, x_{n}$ : $C_{n} \vdash t_{i}: D$ for $i=1,2$, and the type $A \equiv C_{1} \multimap \cdots C_{n} \multimap D$ is $e \Pi_{1}$ (not necessarily closed). Then there is a term if $b$ then $t_{1}$ else $t_{2}$ such that

$$
b: \mathbf{B}, x_{1}: C_{1}, \ldots, x_{n}: C_{n} \vdash \text { if } b \text { then } t_{1} \text { else } t_{2}: D,
$$

where (if true then $t_{1}$ else $t_{2}$ ) $\longrightarrow t_{1}$ and (if false then $t_{1}$ else $t_{2}$ ) $\longrightarrow t_{2}$.

Proof. Define if $b$ then $t$ else $u \equiv$ fst $_{\forall \boldsymbol{\alpha} . A}(b(\lambda \boldsymbol{x} . t)(\lambda \boldsymbol{x} . u)) \boldsymbol{x}$, where $\boldsymbol{x}$ abbreviates $x_{1}, \ldots, x_{n}$ and $\forall \boldsymbol{\alpha} . A$ is the universal closure of $A$. This term can be typed as required; $\lambda \boldsymbol{x}$.t and $\lambda \boldsymbol{x}$.u have type $\forall \boldsymbol{\alpha}$. $A$, thus $b(\lambda \boldsymbol{x} . t)(\lambda \boldsymbol{x} . u)$ has type $\forall \boldsymbol{\alpha} . A \otimes$ $\forall \boldsymbol{\alpha}$.A, to which the projection fst $_{\forall \boldsymbol{\alpha} . A}$ applies. The rest is obvious.

Fix a quantifier-free type $A$ of size $k$, built from a single type variable $\alpha$. A long normal form of type $A$ is a term $t$ such that $\vdash t: A$ has a derivation in which all identity axioms are atomic, i.e., of the form $x: \alpha \vdash x: \alpha$. It is clear that every long normal form $t$ of type $A$ has size bounded by $k$, and we may assume that all variables occurring in it are from a fixed set of variables $\left\{x_{1}, \ldots, x_{k}\right\}$ (due to $\alpha$-equivalence). Therefore, $t$ can be written as a word in $\{0,1\}^{n}$, where $n=O(k \log k)$. Since $\{0,1\}^{n}$ can in turn be represented by $\mathbf{B}^{n}$, there must be a function \lceil\rceil which maps a given term $u$ of size bounded by $k$ into a term $\lceil u\rceil$ of type $\mathbf{B}^{n}$. Furthermore, as a consequence of Theorem 2, we can associate to this coding two terms abs, app : $\mathbf{B}^{n} \multimap \mathbf{B}^{n} \multimap \mathbf{B}^{n}$ which satisfy

$$
\begin{aligned}
& \text { abs }\lceil y\rceil\lceil\rceil \longrightarrow *\lceil\lambda y . t\rceil \text {, if }|\lambda y . t| \leq k \text { and } y \in\left\{x_{1}, \ldots, x_{k}\right\} \text {; } \\
& \operatorname{app}\lceil t\rceil\lceil u\rceil \longrightarrow{ }^{*}\lceil t u\rceil, \quad \text { if }|t u| \leq k .
\end{aligned}
$$

We now show that the coding function \lceil\rceil can be internalized in IMLL, as far as the long normal forms of a fixed type $A$ is concerned. For each subtype $B$ of $A$, define $\sigma_{B}(t)$ and $\tau_{B}(t)$ as follows:

$$
\begin{aligned}
\sigma_{\alpha}(t) & \equiv t & \sigma_{B-C}(t) & \equiv \operatorname{abs}\lceil y\rceil \sigma_{C}\left(t \tau_{B}(\lceil y\rceil)\right) ; \\
\tau_{\alpha}(t) & \equiv t & \tau_{B-C}(t) & \equiv \lambda z \cdot \tau_{C}\left(\operatorname{app} t \sigma_{B}(z)\right) ;
\end{aligned}
$$

where $y$ is from $\left\{x_{1}, \ldots, x_{k}\right\}$ and fresh, in the sense that $\lceil y\rceil$ does not occur in $t$. The term $\sigma_{B}(t)$ has type $\mathbf{B}^{n}$ whenever $t$ has type $B\left[\mathbf{B}^{n} / \alpha\right]$, and $\tau_{B}(t)$ has type $B\left[\mathbf{B}^{n} / \alpha\right]$ whenever $t$ has type $\mathbf{B}^{n}$. Finally, let $\operatorname{code}_{A} \equiv \lambda x \cdot \sigma_{A}(x)$ : $A\left[\mathbf{B}^{n} / \alpha\right] \multimap \mathbf{B}^{n}$.

Lemma 2 (Internal Coding). Let $A$ be as above. For each closed long normal form $t$ of type $A, \operatorname{code}_{A}(t) \longrightarrow\lceil\mid t\rceil$. 
For example, let $A_{1}$ be $((\alpha \multimap \alpha) \multimap \alpha) \multimap(\alpha \multimap \alpha) \multimap \alpha$, which has two long normal forms $t_{1} \equiv \lambda F f . f(F(\lambda y . y))$ and $\left.t_{2} \equiv \lambda F f . F(\lambda y . f y)\right)$. Then code A $_{A_{1}}$ is defined as follows:

$$
\begin{aligned}
\tau_{\alpha-\circ \alpha}(\lceil f\rceil) & \equiv \lambda x \cdot \operatorname{app}\lceil f\rceil x ; \\
\tau_{(\alpha-\alpha \alpha)-\alpha \alpha}(\lceil F\rceil) & \equiv \lambda g \cdot \operatorname{app}\lceil F\rceil(\operatorname{abs}\lceil y\rceil(g\lceil y\rceil)) ; \\
\operatorname{code}_{A_{1}} & \equiv \lambda z \cdot \operatorname{abs}\lceil F\rceil\left(\operatorname{abs}\lceil f\rceil\left(z \tau_{(\alpha-\circ \alpha)-\alpha \alpha}(\lceil F\rceil) \tau_{\alpha-\circ \alpha}(\lceil f\rceil)\right)\right) .
\end{aligned}
$$

It is easy to check that $\operatorname{code}_{F}\left(t_{i}\right)$ reduces to $\left\lceil t_{i}\right\rceil$ for $i=1,2$.

Theorem 3 ( $\Pi_{1}$-Contraction). Let $A$ be a closed $\Pi_{1}$ type which is inhabited. Then there is a contraction map $\operatorname{cntr}_{A}: A \multimap A \otimes A$ such that for any normal form $t$ of type $A, \operatorname{cntr}_{A}(t) \longrightarrow t^{\prime} \otimes t^{\prime}$, where $t^{\prime}$ is a long normal form $\eta$-equivalent to $t$.

Proof. Without loss of generality, we may assume that $A$ is free from $\otimes$ and $\mathbf{1}$. Let $A^{-}$be obtained from $A$ by replacing all subtypes $\forall \beta . C$ by $C[\alpha / \beta]$ for a fixed variable $\alpha$. Then, there is a canonical map iso $A: A \multimap A^{-}[D / \alpha]$ for any $D$ which preserves the structure of terms up to $\eta$-equivalence. By applying Lemma 2 to the type $A^{-}$we obtain a coding map code $A^{-}: A^{-}\left[\mathbf{B}^{n} / \alpha\right] \multimap \mathbf{B}^{n}$.

Let $t_{1}, \ldots, t_{l}$ be the long normal forms of type $A$. By using the conditional in Lemma 1 several times, we can build a term copy $_{A}: \mathbf{B}^{n} \multimap A \otimes A$ which satisfies

$$
\begin{aligned}
& \operatorname{copy}_{A}(u) \longrightarrow{ }^{*} t_{i} \otimes t_{i}, \quad \text { if } u \equiv\left\lceil t_{i}\right\rceil \text {; } \\
& \longrightarrow^{*} t_{1} \otimes t_{1} \text {, otherwise. }
\end{aligned}
$$

Finally, define $\operatorname{cntr}_{A} \equiv \operatorname{copy}_{A} \circ \operatorname{code}_{A^{-}} \circ$ iso $_{A}$.

\section{Additives as Nondeterminism}

\subsection{Additive slices and nondeterministic cut-elimination}

We now move on to the multiplicative additive fragment of Linear Logic. We again confine ourselves to the intuitionistic fragment IMALL, and furthermore, we only consider $\&$ as the additive connective, although $\oplus$ could be added harmlessly. ${ }^{4}$

The terms of IMALL are defined analogously to the terms of IMLL, but we have in addition: (i) if $t$ and $u$ are terms and $F V(t)=F V(u)$, then so is $\langle t, u\rangle$; (ii) if $t$ is a term, then so are $\pi_{1}(t)$ and $\pi_{2}(t)$. The type assignment rules are extended with:

$$
\frac{\Gamma \vdash t_{1}: A_{1} \quad \Gamma \vdash t_{2}: A_{2}}{\Gamma \vdash\left\langle t_{1}, t_{2}\right\rangle: A_{1} \& A_{2}} \quad \frac{x: A_{i}, \Gamma \vdash t: C}{y: A_{1} \& A_{2}, \Gamma \vdash t\left[\pi_{i}(y) / x\right]: C} i=1,2,
$$

\footnotetext{
${ }^{4}$ However, we have to be careful when considering the classical system, which is not confluent as it stands. It could be overcome by adopting Tortora's proofnet syntax with generalized \& boxes, which enjoys confluence [dF03]; see also [MR02].
} 
and the reduction rules are extended with $\pi_{i}\left\langle t_{1}, t_{2}\right\rangle \longrightarrow t_{i}$, for $i=1,2$.

Note that some reductions cause duplication (e.g. $(\lambda x .\langle x, x\rangle) t \longrightarrow\langle t, t\rangle)$, thus cut-elimination in IMALL is no more in linear steps. ${ }^{5}$ However, duplication can be avoided by computing each component of $\left\langle t_{1}, t_{2}\right\rangle$ separately. To formalize this idea, we recall the notion of slice [Gir87].

Definition 2 (Slices). A slice of a term $t$ is obtained by applying the following operation to $t$ as much as possible: $\langle u, v\rangle \mapsto\langle u\rangle_{1}$, or $\langle u, v\rangle \mapsto\langle v\rangle_{2}$.

We say that two slices $t$ and $u$ (of possibly different terms) are compatible if there is no context (i.e. a term with a hole) $\Phi$ such that $t \equiv \Phi\left[\left\langle t^{\prime}\right\rangle_{i}\right], u \equiv \Phi\left[\left\langle u^{\prime}\right\rangle_{j}\right]$, and $i \neq j$.

Lemma 3 (Slicewise checking). Two terms t and $u$ are equivalent if and only if for every compatible pair $\left(t^{\prime}, u^{\prime}\right)$ of slices of $t$ and $u$, we have $t^{\prime} \equiv u^{\prime}$.

The reduction rules are naturally adapted for slices:

$$
(\lambda x . t) u \stackrel{s l}{\longrightarrow} t[u / x] \quad \pi_{i}\langle t\rangle_{i} \stackrel{s l}{\longrightarrow} t \quad \pi_{i}\langle t\rangle_{j} \stackrel{s l}{\longrightarrow} \text { fail, if } i \neq j .
$$

Lemma 4 (Pullback). Let $t \longrightarrow^{*} u$ and $u^{\prime}$ be a slice of $u$. Then there is a unique slice $t^{\prime}$ of $t$ such that $t^{\prime} \stackrel{s l}{\longrightarrow} u^{\prime}$.

Proof. See the following diagrams
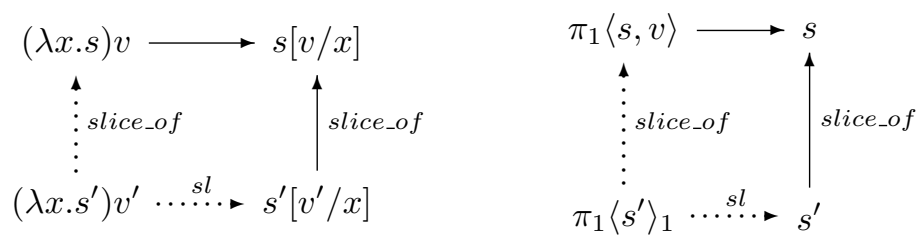

Note that there are exponentially many slices for a given term, but once a slice has been chosen, the computation afterwards can be done in linear steps, thus in quadratic time, since each slice is entirely a linear term. We therefore have a nondeterministic polynomial time cut-elimination procedure, viewing the slicing operation in Definition 2 as a nondeterministic reduction rule. Lemma 3 states that the equivalence of two normal forms can be checked slicewise, and Lemma 4 assures that every slice of a normal form can be obtained by the above nondeterministic procedure. Hence we may conclude that the cut-elimination problem for IMALL is in coNP.

\footnotetext{
5 There is a linear step cut-elimination procedure for terms (proofnets) of lazy types, i.e., those which do not contain positive occurrences of \& and negative occurrences of $\forall$.
} 


\subsection{Encoding a coNP-complete Problem}

Now we show that the following conP-complete problem is logspace reducible to CEP for IMALL:

Logical Equivalence Problem: Given two boolean formulas, are they logically equivalent? (cf. [GJ78])

By Theorem 2, every boolean formula $C$ with $n$ variables can be translated into a term $t_{C}$ of type $\mathbf{B}^{(n)} \multimap \mathbf{B}$ in $O(\log |C|)$ space. For each $1 \leq k \leq n$, let

$$
\operatorname{ta}_{k} \equiv \lambda f . \lambda x_{1} \cdots x_{k-1} \cdot\left\langle f \text { true } x_{1} \cdots x_{k-1}, f \text { false } x_{1} \cdots x_{k-1}\right\rangle,
$$

which is of type $\forall \alpha .\left(\mathbf{B}^{(k)} \multimap \alpha\right) \multimap\left(\mathbf{B}^{(k-1)} \multimap \alpha \& \alpha\right)$, and define ta $\left(t_{C}\right)$ by

$$
\operatorname{ta}\left(t_{C}\right) \equiv \operatorname{ta}_{1}\left(\cdots\left(\operatorname{ta}_{n} t_{C}\right) \cdots\right): \underbrace{\mathbf{B} \& \cdots \& \mathbf{B}}_{2^{n} \text { times }} .
$$

It is clear that the term $\operatorname{ta}\left(t_{C}\right)$ can be built from $t_{C}$ with the help of a counter of size $O(\log n)$.

The normal form of $\operatorname{ta}\left(t_{C}\right)$ consists of $2^{n}$ boolean values, each of which corresponds to a 'truth assignment' to the formula $C$. For example, ta(or) reduces to $\langle\langle$ or true true, or true false $\rangle$, $\langle$ or false true, or false false $\rangle\rangle$,

and thus to $\langle\langle$ true, true $\rangle,\langle$ true, false $\rangle\rangle$.

Therefore, two formulas $C$ and $D$ with $n$ variables are logically equivalent if and only if $\operatorname{ta}\left(t_{C}\right)$ and $\operatorname{ta}\left(t_{D}\right)$ reduce to the same normal form.

Theorem 4 (coNP-completeness of IMALL). The cut-elimination problem for IMALL is coNP-complete.

Remark 1. We do not claim that the complexity of MALL is conP. What we have shown is that a specific problem, CEP for MALL, is complete for coNP. If we had considered the complement of CEP, then the result would have been NP-completeness. Likewise, we could obtain a $\mathcal{C}$-completeness result for any class $\mathcal{C}$ in the polynomial time hierarchy by complicating the problem more and more.

However, we do claim that additives have something to do with nondeterminism, as they provide a notion of nondeterminsitic cut-elimination, as well as a very natural coding of nondeterministic Turing machine computation.

\section{Multiplicative Light Linear Logic and 2EXPTIME}

In this section, we show that the intuitionistic multiplicative fragment IMLLL of Light Linear Logic is already expressive enough to represent all polynomial time functions; it needs neither additives (as in [Gir98]) nor unrestricted weakening (as in [Asp98]).

Since our concern is not normalization but representation, we do not need to introduce a proper term calculus with the polynomial time normalization 
property (see [Asp98] and [Ter01] for such term calculi). We rather use the standard $\lambda$-calculus and think of IMLLL as a typing system for it.

The type assignment rules of IMLLL are those of IMLL with the following:

$\frac{x: B \vdash t: A}{x: ! B \vdash t: ! A} \quad \frac{\Gamma \vdash t: C}{x: ! B, \Gamma \vdash t: C} \quad \frac{x: ! A, y: ! A, \Gamma \vdash t: C}{z: ! A, \Gamma \vdash t[z / x, z / y]: C} \quad \frac{\bar{x}: \bar{A}, \bar{y}: \bar{B} \vdash t: C}{\bar{x}: \overline{! A}, \bar{y}: \overline{\S B} \vdash t: \S C}$

where $x: B$ may be absent in the first rule. Define $\mathbf{W}$ to be $\forall \alpha . !(\mathbf{B} \multimap \alpha \multimap$ $\alpha) \multimap \S(\alpha \multimap \alpha)$. Then each word $w=i_{1} \cdots i_{n}$, where $n \geq 0$ and $i_{k} \in\{0,1\}$, is represented by $\underline{w} \equiv \lambda c x . c i_{1} \circ \cdots \circ c i_{n}(x): \mathbf{W}$, where $i_{k}$ is false if $i_{k}=0$, and is true if $i_{k}=1$. A function $f:\{0,1\}^{*} \longrightarrow\{0,1\}^{*}$ is represented by a term $t$ if $f(w)=v \Longleftrightarrow t \underline{w} \longrightarrow^{*} \underline{v}$.

Simulation of polynomial time Turing machines in Light Linear Logic [Gir98,AR02] consists of two parts; one for coding of polynomials and the other for simulation of one-step transition (as well as initialization and output extraction). Since the former is already additive-free in [Gir98], we focus on the latter here.

Let $M$ be a Turing machine with two symbols ${ }^{6}$ and $2^{n}$ states, and let

$$
\delta: \text { Symbols } \times \text { States } \longrightarrow \text { Symbols } \times \text { States } \times\{\text { left }, \text { right }\}
$$

be the associated instruction function. A configuration of $M$ can be specified by a triple $\left\langle w_{1}, w_{2}, q\right\rangle$, where the stack $w_{1} \in\{0,1\}^{*}$ describes the non-blank part of the tape to the left of the head, the stack $w_{2} \in\{0,1\}^{*}$ describes the non-blank part of the tape to the right of the head, and $q<2^{n}$ denotes the current state. By convention, $w_{1}$ is written in the reverse order, and $w_{1}$ includes the content of the cell currently scanned.

The configurations are represented by terms of type $I D\left[\mathbf{B}^{n}\right]$, where $I D[A]$ is defined by $I D[A] \equiv \forall \alpha . !(\mathbf{B} \multimap \alpha \multimap \alpha) \multimap \S(\alpha \multimap \alpha \multimap(\alpha \otimes \alpha \otimes A))$. Note that $I D[A]$ is a generalization of $\mathbf{W}$, which allows to encode two words and an additional datum of type $A$ into one term. For example, the configuration $\langle 010,11,7\rangle$ is represented by

$$
\underline{\langle 010,11, q\rangle} \equiv \lambda c . \lambda x_{1} x_{2} \cdot\left(c \underline{0} \circ c \underline{1} \circ c \underline{0}\left(x_{1}\right)\right) \otimes\left(c \underline{1} \circ c \underline{1}\left(x_{2}\right)\right) \otimes \underline{q},
$$

where $\underline{q}$ is a term of type $\mathbf{B}^{n}$ coding $q \in$ States.

To simulate one-step transition, it is convenient to divide it into two parts: the decomposition part and the combination part.

Lemma 5 (Decomposition). There is a term dec : ID $\left[\mathbf{B}^{n}\right]-I D\left[\mathbf{B} \otimes \mathbf{B} \otimes \mathbf{B}^{n}\right]$ such that for any configuration $\left\langle i_{1} \cdots i_{n}, j_{1} \cdots j_{m}, q\right\rangle$,

$$
\operatorname{dec} \underline{\left\langle i_{1} \cdots i_{n}, j_{1} \cdots j_{m}, q\right\rangle} \longrightarrow \underline{\left.* i_{2} \cdots i_{n} 0, j_{2} \cdots j_{m} 0, i_{1}, j_{1}, q\right\rangle} .
$$

\footnotetext{
${ }^{6}$ Although more than two symbols are required in general, we describe the two symbols version here for simplicity. The extension is straightforward.
} 
Proof. Define dec $(\operatorname{conf})$ to be $\lambda c . G(\operatorname{conf} F(c))$, where the 'step' function $F$ and the 'basis' function $G$ are defined as follows;

$$
\begin{aligned}
& F(c) \equiv \lambda b_{1} \cdot \lambda b_{2} \otimes w \cdot\left(b_{1} \otimes\left(c b_{2} w\right)\right) \\
& \begin{array}{l}
G(y) \equiv \lambda x_{1} x_{2} \cdot \text { let }\left(y\left(\underline{0} \otimes x_{1}\right)\left(\underline{0} \otimes x_{2}\right)\right) \text { be }\left(i_{1} \otimes w_{1}\right) \otimes\left(j_{1} \otimes w_{2}\right) \otimes q \text { in } \\
\left(w_{1} \otimes w_{2} \otimes i_{1} \otimes j_{1} \otimes q\right)
\end{array} \\
& \begin{array}{l}
c: B \multimap \alpha \multimap \alpha \vdash F(c): \mathbf{B} \multimap D \multimap D \\
y: D \multimap D \multimap D \otimes D \otimes \mathbf{B}^{n} \vdash G(y): \alpha \multimap \alpha \multimap\left(\alpha \otimes \alpha \otimes \mathbf{B} \otimes \mathbf{B} \otimes \mathbf{B}^{n}\right) .
\end{array}
\end{aligned}
$$

Here, $D$ stands for $\mathbf{B} \otimes \alpha$. The use of $F$ may be illustrated by

$$
\left(F(c) \underline{i_{1}}\right) \circ \cdots \circ\left(F(c) \underline{i_{n}}\right)(\underline{0} \otimes x) \longrightarrow \underline{i}^{*} \otimes\left(c \underline{i_{2}} \circ \cdots \circ c \underline{i_{n}} \circ c \underline{0}(x)\right): D,
$$

while $G$ plays the roles of initialization and rearrangement of the output.

Lemma 6 (Combination). There is a term com : ID $\left[\mathbf{B} \otimes \mathbf{B} \otimes \mathbf{B}^{n}\right] \multimap I D\left[\mathbf{B}^{n}\right]$ such that for any $\left\langle w_{1}, w_{2}, i_{1}, i_{2}, q\right\rangle$ with $\delta\left(i_{1}, q\right)=\left(s, q^{\prime}, m\right)$,

$$
\begin{aligned}
& \operatorname{com}\left\langle w_{1}, w_{2}, i_{1}, i_{2}, q\right\rangle \longrightarrow\left\langle w_{1}, s i_{2} w_{2}, q^{\prime}\right\rangle \text {, if } m=\text { left; } \\
& \longrightarrow * \overline{\left\langle i_{2} s w_{1}, w_{2}, q^{\prime}\right\rangle}, \text { if } m=\text { right. }
\end{aligned}
$$

Proof. Let left $\equiv$ true and right $\equiv$ false. By Theorem 2, there is a term delta such that delta $\underline{i_{1}} q$ reduces to $\underline{s} \otimes \underline{q^{\prime}} \otimes \underline{m}$ when $\delta\left(i_{1}, q\right)=\left(s, q^{\prime}, m\right)$. Now the key trick is to use the boolean value $\underline{m}$ as "switcher." Observe that $\underline{m s} \underline{i_{2}}$ reduces to $\underline{s} \otimes \underline{i_{2}}$ $\left(\underline{i_{2}} \otimes \underline{s}\right)$ and $\underline{m} \underline{w_{1}} w_{2}$ reduces to $\underline{w_{1}} \otimes \underline{w_{2}}\left(\underline{w_{2}} \otimes \underline{w_{1}}\right)$ when $m$ is left (right) - thus $m$ can be used to determine on which side of the tape we push symbols, and in what order they are pushed.

Formally, let $\mathrm{cntr}^{3}: \mathbf{B} \multimap \mathbf{B}^{3}$ be a generalized contraction which produces three copies of a given boolean value, and define $G\left(m, w_{1}, w_{2}, i_{2}, s, c_{1}, c_{2}\right)$ to be

let $\operatorname{cntr}^{3}(m)$ be $m_{1} \otimes m_{2} \otimes m_{3}$ in (let $m_{1} s i_{2}$ be $j_{1} \otimes j_{2}$ in

(let $m_{2} w_{1} w_{2}$ be $v_{1} \otimes v_{2}$ in $\left.m_{3} v_{1}\left(c_{1} j_{1} \circ c_{2} j_{2}\left(v_{2}\right)\right)\right)$ ),

which is of type

$m: \mathbf{B}, w_{1}: \alpha, w_{2}: \alpha, i_{2}: \mathbf{B}, s: \mathbf{B}, c_{1}: \mathbf{B} \multimap \alpha \multimap \alpha, c_{2}: \mathbf{B} \multimap \alpha \multimap \alpha \vdash$ $G\left(m, w_{1}, w_{2}, i_{2}, s, c_{1}, c_{2}\right): \alpha \otimes \alpha$. Then, depending on the value of $m$, it reduces as follows:

$G\left(\right.$ true $\left., w_{1}, w_{2}, i_{2}, s, c, c\right) \longrightarrow w_{1} \otimes\left(c s \circ c i_{2}\left(w_{2}\right)\right) ;$

$G\left(\right.$ false, $\left.w_{1}, w_{2}, i_{2}, s, c, c\right) \longrightarrow\left(c i_{2} \circ c s\left(w_{1}\right)\right) \otimes w_{2}$.

Finally, the term com is defined to be

$\lambda z . \lambda c x_{1} x_{2}$. let $z c x_{1} x_{2}$ be $w_{1} \otimes w_{2} \otimes i_{1} \otimes i_{2} \otimes q$ in

(let delta $i_{1} q$ be $s \otimes q^{\prime} \otimes m$ in $\left.G\left(m, w_{1}, w_{2}, i_{2}, s, c, c\right) \otimes q^{\prime}\right)$.

Although the 'cons' variable $c$ is used three times in com, it does not matter since it is assigned a type ! $(\mathbf{B} \multimap \alpha \multimap \alpha)$.

The desired one-step transition function is obtained by composing dec and com. 
Theorem 5 (IMLLL represents PTIME functions). A function $f:\{0,1\}^{*} \longrightarrow$ $\{0,1\}^{*}$ is computable in DTIME $\left[n^{k}\right]$ if and only if it is represented by an IMLLL term $t$ of type $\mathbf{W} \multimap \S^{d} \mathbf{W}$, where $d=O(\log k)$.

In general, cut-elimination in Light Affine Logic, hence in IMLLL, requires of time $O\left(s^{2^{d+1}}\right)$, where $s$ is the size of a proof and $d$ is its depth, which counts the nesting of ! and $\S$ inferences. The reason why we have a characterization of PTIME above is that we consider a fixed program $t$, so all the terms $t \underline{w}$ to be evaluated have a fixed depth. On the other hand, CEP allows the depth to vary, hence we get a characterization of doubly-exponential time as in [NM02].

Theorem 6 (2EXPTIME-completeness of IMLLL). The cut-elimination problem for IMLLL is complete for $2 \mathrm{EXPTIME}=\bigcup_{k}$ DTIME $\left[2^{2^{n^{k}}}\right]$.

\section{Multiplicative Soft Linear Logic and EXPTIME}

In this section, we show that the intuitionistic mulitplicative fragment IMSLL of Soft Linear Logic is expressive enough to represent all polynomial time functions, as conjectured by Lafont [Laf01]. As before, we do not introduce a term calculus for IMSLL, thinking of it as a type assignment system for the standard $\lambda$ calculus.

The type assignment rules of IMSLL are those of IMLL with the following:

$$
\frac{x_{1}: B_{1}, \ldots, x_{m}: B_{m} \vdash t: A}{x_{1}: ! B_{1}, \ldots, x_{m}: ! B_{m} \vdash t: ! A} m \geq 0 \quad \frac{x_{1}: A, \ldots, x_{n}: A, \Gamma \vdash t: C}{z: ! A, \Gamma \vdash t\left[z / x_{1}, \ldots, z / x_{n}\right]: C} n \geq 0
$$

The former is called soft promotion and the latter is called multiplexing. A term which can be typed without multiplexing is called generic. Note that every generic term is a linear $\lambda$-term.

The policy of MSLL programming is to write each program in a generic way; multiplexing (i.e. duplication) is used only in data. Due to this restriction, simulation of Turing machines is more sophisticated than before. Let $M$ and $\delta$ be as before. Let $I D_{k}[A]$ be $\forall \alpha . !(\mathbf{B} \multimap \alpha \multimap \alpha) \multimap\left((\alpha \multimap \alpha)^{k} \otimes A\right)$. Each term of type $I D_{k}[A]$ encodes $k$ words as well as an element of type $A$. For instance, the configuration $\langle 010,11,7\rangle$ is represented by

$$
\underline{\langle 010,11,7\rangle} \equiv \lambda c .(c \underline{0} \circ c \underline{1} \circ c \underline{0}) \otimes(c \underline{1} \circ c \underline{1}) \otimes \underline{7}: I D_{2}\left[\mathbf{B}^{n}\right] .
$$

Lemma 7 (Decomposition). For every $k \geq 1$, there exists a generic term dec of type $I D_{k}\left[\mathbf{B}^{n}\right] \multimap I D_{2 k}\left[\mathbf{B} \otimes \mathbf{B}^{n}\right]$ such that for any $\left\langle i_{1} w_{1}, \ldots, i_{k} w_{k}, q\right\rangle \in$ $\left(\{0,1\}^{+}\right)^{k} \times 2^{n}$,

$$
\operatorname{dec}\left\langle i_{1} w_{1}, \ldots, i_{k} w_{k}, q\right\rangle \longrightarrow\left\langle w_{1}, \ldots, w_{k}, i_{1}, \ldots, i_{k}, i_{1}, q\right\rangle .
$$

Note that the output contains two occurrences of $i_{1}$; the first is a word of length 1 which will be thrown away, while the second is a boolean value which will be used as the current symbol in the next combination part. 


\begin{tabular}{|ccccccc|}
\hline left tape & $\downarrow$ & & right tape & left tape & $\downarrow$ & right tape \\
$w_{1}$ & $i_{1}$ & $i_{2}$ & $w_{2}$ & $w_{1}$ & $1 i_{2}$ & $w_{2}$ \\
stocks of 0 & & & stocks of $1 \Longrightarrow$ & stocks of 0 & & stocks of 1 \\
$w_{3}$ & 0 & 1 & $w_{4}$ & $w_{3}$ & 0 & $w_{4}$ \\
garbages & & & & garbages & & \\
$w_{5}$ & $i_{5}$ & & & $w_{5}$ & $i_{5} i_{1}$ & \\
\hline
\end{tabular}

Fig. 1. "Write 1 and move right" ( $\downarrow$ indicates the head position)

Proof. Consider the case $k=1$. The term dec is defined to be $\lambda z \cdot \lambda c . \lambda z \otimes$ $q .(z F(c)(i d \otimes i d \otimes \underline{0})) \otimes q$, where the step function $F$ is defined by

$$
\begin{aligned}
& \left.F(c) \equiv \lambda b \text {.let } \operatorname{cntr}(b) \text { be } b_{1} \otimes b_{2} \text { in }\left(\lambda g \otimes h \otimes e . f s t\left((h \circ g) \otimes c b_{1} \otimes b_{2}\right) \otimes e\right)\right) \\
& c: \mathbf{B} \multimap \alpha \multimap \alpha \vdash F(c): \mathbf{B} \multimap\left((\alpha \multimap \alpha)^{2} \otimes \mathbf{B}\right) \multimap\left((\alpha \multimap \alpha)^{2} \otimes \mathbf{B}\right) .
\end{aligned}
$$

The behavior of $F$ is illustrated as follows;

$$
\left(F(c) \underline{i_{1}}\right) \circ \cdots \circ\left(F(c) \underline{i_{n}}\right)(i d \otimes i d \otimes \underline{0}) \longrightarrow\left(c \underline{i_{2}} \circ \cdots \circ c \underline{i_{n}}\right) \otimes c \underline{i_{1}} \otimes c \underline{i_{1}} .
$$

The case $k \geq 2$ is similar, except that we remove all redundant boolean values $i_{2}, \ldots, i_{k}$ by weakening for $\mathbf{B}$.

Now let us move on to the combination part. Due to the genericity restriction, we face two difficulties: (i) we cannot create a new tape cell, since the 'cons' variable $c$ of type !(B $\multimap \alpha \multimap \alpha)$ cannot be used twice; (ii) we cannot simply remove an unnecessary tape cell of type $\alpha \multimap \alpha$, since we do not have weakening for the open type $\alpha \multimap \alpha$. To resolve the first difficulty, we prepare two additional stacks which are filled with $\underline{0}$ 's and $\underline{1}$ 's respectively, and instead of creating a new cell, we pick one from these two stacks according to the instruction $\delta$. To resolve the second difficulty, we further prepare a 'garbage' stack where unneccesary tape cells are collected. Thus we associate five stacks in total with a configuration. The transition corresponding to "write 1 and move right" is illustrated in Figure 1.

Lemma 8 (Combination). There is a generic term com of type $I D_{10}[\mathbf{B} \otimes$ $\left.\mathbf{B}^{n}\right] \multimap I D_{5}\left[\mathbf{B}^{n}\right]$ such that for any $\left\langle w_{1}, \ldots, w_{5}, i_{1}, \ldots, i_{5}, b, q\right\rangle \in\left(\{0,1\}^{+}\right)^{5} \times$ $\{0,1\}^{5} \times\{0,1\} \times 2^{n}$ with $\delta(b, q)=\left(s, q^{\prime}, m\right)$,

$$
\begin{aligned}
& \operatorname{com}\left\langle w_{1}, \ldots, w_{5}, i_{1}, i_{2}, 0,1, i_{5}, b, q\right\rangle \\
& \longrightarrow^{*}\left\langle w_{1}, 0 i_{2} w_{2}, w_{3}, 1 w_{4}, i_{1} i_{5} w_{5}, q^{\prime}\right\rangle \quad \text { if } s=0 \text { and } m=\text { left; } \\
& \longrightarrow^{*} \overline{\left\langle w_{1}, 1 i_{2} w_{2}, 0 w_{3}, w_{4}, i_{1} i_{5} w_{5}, q^{\prime}\right\rangle} \quad \text { if } s=1 \text { and } m=\text { left; } \\
& \longrightarrow{ }^{*} \overline{\left\langle i_{2} 0 w_{1}, w_{2}, w_{3}, 1 w_{4}, i_{1} i_{5} w_{5}, q^{\prime}\right\rangle} \quad \text { if } s=0 \text { and } m=\text { right; }
\end{aligned}
$$

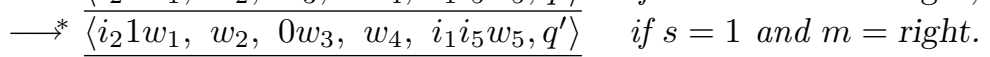

Keep in mind that the third and the fourth stacks are to be filled with $\underline{0}$ 's and $\underline{1}$ 's, so that we always find $\underline{0}$ and $\underline{1}$ at positions $i_{3}$ and $i_{4}$, respectively. 
Proof. As before, there is a term delta such that delta $\underline{b} \underline{q}$ reduces to $\underline{s} \otimes \underline{q^{\prime}} \otimes \underline{m}$ when $\delta(b, q)=\left(s, q^{\prime}, m\right)$. Define 1Right by

$$
\begin{aligned}
& 1 \text { Right } \equiv\left(i_{2} \circ i_{4} \circ w_{1}\right) \otimes w_{2} \otimes\left(i_{3} \circ w_{3}\right) \otimes w_{4} \otimes\left(i_{1} \circ i_{5} \circ w_{5}\right) \\
& w_{1}: \alpha \multimap \alpha, \ldots, w_{5}: \alpha \multimap \alpha, i_{1}: \alpha \multimap \alpha, \ldots, i_{5}: \alpha \multimap \alpha \vdash 1 \text { Right }:(\alpha \multimap \alpha)^{5},
\end{aligned}
$$

which corresponds to the case $s=1$ and $m=$ right (see Figure 1) and gives five stacks as output. OLeft, 1Left and ORight are defined analogously. By using conditionals in Lemma 1 three times, we obtain

$$
\begin{aligned}
& G\left(m, s, w_{1}, \ldots, w_{5}, i_{1}, \ldots, i_{5}\right) \equiv\left(\begin{array}{r}
\text { if } m \text { then if } s \text { then 0Left else 1Left } \\
\text { then if } s \text { then 0Right else 1Right }
\end{array}\right) \\
& \text { com } \equiv \lambda z . \lambda c . \text { let } z c \text { be } w_{1} \otimes \cdots \otimes w_{5} \otimes i_{1} \otimes \cdots \otimes i_{5} \otimes b \otimes q \text { in }
\end{aligned}
$$

(let delta $b q$ be $s \otimes q^{\prime} \otimes m$ in $\left.G\left(m, s, w_{1}, \ldots, w_{5}, i_{1}, \ldots, i_{5}\right) \otimes q^{\prime}\right)$.

The rest of coding is basically the same as in [Laf01] except the initialization part, where we need to fill two stacks with $\underline{0}$ 's and $\underline{1}$ 's. As in [Laf01], we have no idea how to extract a single word as output from the final configuration consisting of five stacks. Instead, we can extract the boolean value which tells us whether the final configuration is accepting or not. Thus the representation theorem below is stated in terms of languages rather than functions in general. Furthermore, due to the genericity restriction, we need to relax the definition of representation slightly. The set $\{0,1\}^{*}$ is represented by $\mathbf{W} \equiv \forall \alpha . !(\mathbf{B} \multimap \alpha \multimap \alpha) \multimap \alpha \multimap \alpha$. We say that a language $X \subseteq\{0,1\}^{*}$ is represented by a term $t: \mathbf{W}^{l} \multimap \mathbf{B}$ if $w \in X \Longleftrightarrow t \underbrace{\underline{w} \cdots \underline{w}}_{l \text { times }} \longrightarrow$ true.

Theorem 7 (IMSLL captures PTIME). A language $X \subseteq\{0,1\}^{*}$ is accepted in

DTIME $\left[n^{k}\right]$ if and only if it is represented by a generic term $t$ of type $\mathbf{W}^{l} \multimap \mathbf{B}$, where $l=O(k)$.

As in the case of IMLLL, the complexity of CEP exceeds polynomial time. A difference is that cut-elimination in IMSLL only requires exponential time $O\left(s^{d+2}\right)$ [Laf01]. Hence we have:

Theorem 8 (EXPTIME-completeness of IMSLL). The cut-elimination problem for IMSLL is complete for EXPTIME under logspace reducibility.

Proof (sketch). Suppose that a language $X$ be accepted by a Turing machine $M$ in time $O\left(2^{n^{k}}\right)$. For each word $w$ of length $n$, the following terms (of suitable types) can be constructed in $O(k \log n)$ space: (1) the Church representation $\underline{w}$ of $w ;(2)$ the term $\exp \left(n^{k}\right)$ of size and depth $O\left(n^{k}\right)$, which reduces to the tally integer $\frac{2^{n^{k}}}{2}$; (3) the term $\mathrm{M}_{n, k}(w, x)$ with two variables $w$ and $x$, which outputs the result of $x$-steps computation on the input $w$, when $w$ is of length $n$ and 
$x$ is of the same type as $\exp \left(n^{k}\right)$. By putting them together, we obtain a term $\mathrm{M}_{n, k}\left(\underline{w}, \exp \left(n^{k}\right)\right)$ which normalizes to true if and only if $w \in X$.

Acknowledgments. We wish to thank Patrick Baillot and Marco Pedicini for very stimulating discussions, and Jean-Yves Girard, Stefano Guerrini, Yves Lafont, Satoshi Matsuoka, Peter Neergaard, Peter Selinger, Izumi Takeuti and Rene Vestergaard for a lot of useful comments.

\section{References}

[Asp98] A. Asperti. Light affine logic. In Proceedings of the Thirteenth Annual IEEE Symposium on Logic in Computer Science, pages 300-308, 1998.

[AR02] A. Asperti and L. Roversi. Intuitionistic light affine logic (proof-nets, normalization complexity, expressive power, programming notation). ACM Transactions on Computational Logic, 3(1):1-39, 2002.

[dF03] L. Tortora de Falco. The additive multiboxes. Annals of Pure and Applied Logic, 120(1):65-102, 2003.

[Gir87] J.-Y. Girard. Linear logic. Theoretical Computer Science, 50:1-102, 1987.

[Gir98] J.-Y. Girard. Light linear logic. Information and Computation, 14(3):175-204, 1998.

[GJ78] M. Garey and D. Johnson. Computers and Intractability: A Guide to the Theory of NP-completeness. Freeman, San Francisco, 1978.

[Hin89] J. R. Hindley. BCK-combinators and linear $\lambda$-terms have types. Theoretical Computer Science, 64:97-105, 1989.

[Lad75] R. E. Ladner. The circuit value problem is logspace complete for P. SIGACT News, 7(1):18-20, 1975.

[Laf01] Y. Lafont. Soft linear logic and polynomial time. Theoretical Computer Science, to appear.

[Lin95] P. D. Lincoln. Deciding provability of linear logic formulas. In Advances in Linear Logic, London Mathematical Society Lecture Notes Series, Volume 222, Cambridge University Press, 1995, 109-122.

[MR02] H. G. Mairson and X. Rival. Proofnets and context semantics for the additives. Computer Science Logic (CSL) 2002, 151-166.

[Mai03] H. G. Mairson. Linear lambda calculus and polynomial time. Journal of Functional Programming, to appear.

[NM02] P. M. Neergaard and H. G. Mairson. LAL is square: representation and expressiveness in light affine logic. Presented at the Fourth International Workshop on Implicit Computational Complexity, 2002.

[Sch01] A. Schubert. The Complexity of $\beta$-Reduction in Low Orders. Typed Lambda Calculi and Applications (TLCA) 2001, 400-414.

[Sta79] R. Statman. The typed $\lambda$-calculus is not elementary recursive. Theoretical Computer Science, 9:73-81, 1979.

[Ter01] K. Terui. Light affine lambda calculus and polytime strong normalization. In Proceedings of the sixteenth annual IEEE symposium on Logic in Computer Science, pages 209-220, 2001. The full version is available at http://research.nii.ac.jp/ terui. 